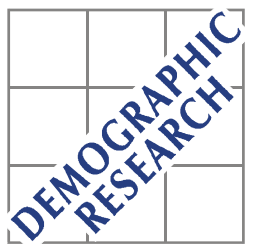

Demographic Research a free, expedited, online journal of peer-reviewed research and commentary in the population sciences published by the Max Planck Institute for Demographic Research Doberaner Strasse 114 D D-18057 Rostock · GERMANY www.demographic-research.org

DEMOGRAPHIC RESEARCH

VOLUME 6, ARTICLE 3, PAGES 49-66

PUBLISHED 29 JANUARY 2002

www.demographic-research.org/Volumes/Vol6/3/

DOI: $10.4054 /$ DemRes.2002.6.3

\title{
On the impact of spatial momentum
}

\section{Robert Schoen}

(C) 2002 Max-Planck-Gesellschaft. 


\section{Table of Contents}

1 Introduction $\quad 50$

$2 \quad$ A simple model with spatial momentum $\quad 51$

3 Decomposing the momentum of a multi-regional 53 population

$4 \quad$ Numerical results from hypothetical populations 56

$5 \quad$ Spatial momentum in Mexico, $1970 \quad 59$

$6 \quad$ Summary and conclusions 61

7 Acknowledgments $\quad 61$

$\begin{array}{ll}\text { Notes } & 62\end{array}$

$\begin{array}{ll}\text { References } & 63\end{array}$

$\begin{array}{lll}\text { A } & \text { Appendix } & 65\end{array}$ 


\title{
On the impact of spatial momentum
}

\author{
Robert Schoen ${ }^{1}$
}

\begin{abstract}
Momentum, the population growth that occurs after a fall in fertility to replacement level, has long been appreciated as a factor in the future population growth of many countries. This paper argues that another aspect of growing populations-their high proportion rural-is also a potential source of significant growth, referring to the additional growth attributable to geographical redistribution as spatial momentum. Using simplifying assumptions, a model for analyzing spatial momentum is developed based on population composition, rates of growth, and levels of interregional migration. Calculations are then done using (i) hypothetical populations exhibiting a range of plausible demographic behavior, and (ii) the population of Mexico, 1970. The results show that spatial momentum can have a substantial impact on ultimate population size under commonly encountered circumstances.
\end{abstract}

1 Department of Sociology, Pennsylvania State University, University Park PA 16802, USA.

Email: schoen@pop.psu.edu 


\section{Introduction}

Population momentum is the amount of population growth that occurs after a fall in fertility to replacement level. The phenomenon was first analyzed by Keyfitz (1971), who showed that growing populations would have substantial increases in size even if they experienced an immediate fall in fertility to replacement. Among contemporary Third World countries, he found that momentum frequently produced growth in the range of 50-70\%. Population momentum is an important factor today, as a recent study by Bongaarts and Bulatao (1999) showed that momentum is likely to be the main cause of future growth in most countries and regions of the world. As analyzed by Preston (1986), Wachter (1988), Kim, Schoen and Sarma (1991), and Kim and Schoen (1993; 1997), momentum has been shown to be a consequence of the young age structure of growing populations, and inseparable from the phenomenon of population aging.

Momentum is generally discussed with respect to age structure alone, but is actually much broader in scope. For example, Schoen (1988: 191) examined the momentum related to changes in the sex composition of a population. Apart from age, the most important characteristic associated with momentum is geographical mobility. Here the term spatial momentum is used to refer to the increase in population size that occurs after a fall in fertility to replacement level that is attributable to the geographical redistribution of the population.

Rogers and Willekens (1978) pioneered the analysis of spatial momentum using multi-regional stable populations that recognized mortality, fertility, and interregional migration. Applying their approach to estimated data, they showed how India's urban fraction would grow substantially during the transition to zero growth, compounding human settlement concerns. Although urban growth in developing countries since 1980 has been slower than anticipated (Brockerhoff 1999), urbanization has continued and is expected to be substantial in the decades ahead (United Nations 1999).

Spatial momentum related to urbanization typically arises from two commonly encountered demographic patterns. The first is that a multi-regional population's rate of natural increase is higher in rural than in urban areas. That generally occurs because of the higher fertility usually found in rural areas and because of their younger age composition, which lowers rural crude death rates [1]. As a result, in the ultimate stationary (zero growth) population, the rural areas have a positive rate of natural increase and the urban areas a negative rate of natural increase. The population as a whole has zero growth because of net migration from rural to urban areas. The second pattern is that the rural areas are over-represented in the initial population compared to the ultimate stationary population. Since most developing countries are urbanizing, that is typically the case. Spatial momentum is the additional growth produced during the 
transition to stationarity by the larger number of persons residing in the faster growing rural areas.

Here we assess spatial momentum's contribution to total growth over a population's transition to zero growth. To begin, we analyze a basic two-region model to identify the principal factors underlying spatial momentum. We then describe how the total momentum of a multi-regional population can be decomposed into components attributable to changes in age structure and to spatial reallocation. Hypothetical populations, with plausible rates covering a range of trajectories to stationarity, are then examined, along with the urban and rural population of Mexico, 1970, in order to quantify the magnitude of the growth attributable to spatial momentum.

\section{A simple model with spatial momentum}

Schoen and Kim (1993) analyzed a basic two region model that did not recognize age. The population projection matrix of that model, $\mathbf{R}$, can be written in the form

$$
\mathbf{R}=\left[\begin{array}{ll}
r_{11} & r_{21} \\
r_{12} & r_{22}
\end{array}\right]
$$

Where $r_{i j}$ represents the contribution of each person in state $i$ to the number of persons in state $\mathrm{j}$ one interval later. The rate matrix underlying $\mathbf{R}$ can be written

$$
\mu_{\mathbf{R}}=\left[\begin{array}{cc}
\rho_{1}-\mathrm{m}_{12} & \mathrm{~m}_{21} \\
\mathrm{~m}_{12} & \rho_{2}-\mathrm{m}_{21}
\end{array}\right]
$$

where $\rho_{\mathrm{j}}$ represents the rate of natural increase (i.e. the birth rate minus the death rate) in state $\mathrm{j}$, and $\mathrm{m}_{\mathrm{ij}}$ represents the rate of interregional transfer from state $\mathrm{i}$ to state $\mathrm{j}$.

The major analytical finding of Schoen and Kim (1993:eq 20) was that the spatial momentum $\left(\Omega_{\mathrm{R}}\right)$ of that model population is given by

$$
\Omega_{\mathrm{R}}-1=-\left(1 / \mathrm{s}_{\mu}\right) \frac{\left\lfloor\left(\rho_{1}-\mathrm{r}_{\mu}\right) \mathrm{U}_{0}+\left(\rho_{2}-\mathrm{r}_{\mu}\right) \mathrm{N}_{0}\right\rfloor}{\mathrm{U}_{0}+\mathrm{N}_{0}}
$$


where $\mathrm{U}_{0}$ and $\mathrm{N}_{0}$ represent, respectively, the initial populations in the urban and rural (non-urban) regions, $r_{\mu}$ represents the dominant eigenvalue of $\boldsymbol{\mu}_{\mathbf{R}}$, and $s_{\mu}$ denotes the subordinate eigenvalue of stationary matrix $\boldsymbol{\mu}_{\mathbf{R}}{ }^{*}=\boldsymbol{\mu}_{\mathbf{R}}-r_{\mu} \mathbf{I}$, where $\mathbf{I}$ is the identity matrix of order 2 . They interpreted that equation as showing that spatial momentum was the product of two factors. The first factor, $-\left(1 / s_{\mu}\right)>0$, reflected the reciprocal of the speed of convergence to stationarity, with slower convergence yielding greater spatial momentum. The second factor, the growth rate of the population immediately after the fall in fertility to replacement, reflected both differential growth by region and the departure of the initial population from the ultimate stationary composition. Schoen and Kim (1993) concluded that spatial momentum led to population growth only when the faster growing state was overrepresented in the initial population, with the magnitude of that growth more sensitive to the speed of convergence to stationarity.

The dominant eigenvalue of a rate matrix is familiar to demographers as Lotka's intrinsic rate of population growth. The subordinate eigenvector is not a commonly encountered quantity, however, nor is the rate of convergence to stationarity a familiar concept. To find a more demographically meaningful expression for spatial momentum, let us turn to a simpler case and assume that $\mathrm{m}_{12}=0$, i.e. that there is no migration from state 1 (urban) to state 2 (rural). With $\mathrm{m}_{12}=0$, our restricted rate matrix $\boldsymbol{\mu}_{\mathbf{R R}}$ can be written as

$$
\boldsymbol{\mu}_{\mathbf{R R}}=\left[\begin{array}{cc}
\rho_{1} & \mathrm{~m}_{21} \\
0 & \rho_{2}-\mathrm{m}_{21}
\end{array}\right]
$$

To avoid instances where the rural population goes to zero, we require that $\left(\rho_{2}\right.$ $\left.\mathrm{m}_{21}\right)>\rho_{1}$. Then $\left(\rho_{2}-\mathrm{m}_{21}\right)$ is the dominant eigenvalue of $\boldsymbol{\mu}_{\mathbf{R R}}$, and $\rho_{1}$ is its subordinate eigenvalue. Applying equation (3), spatial momentum becomes

$$
\Omega_{\mathrm{RR}}=\frac{\mathrm{N}_{0}}{\mathrm{U}_{0}+\mathrm{N}_{0}} \frac{\rho_{2}-\rho_{1}}{\rho_{2}-\rho_{1}-\mathrm{m}_{21}}
$$

Three interpretable elements influencing spatial momentum can be identified in equation (5). They are:

(i) $\mathrm{N}_{0} /\left(\mathrm{U}_{0}+\mathrm{N}_{0}\right)$, the initial proportion rural. The more rural (or less urban) the initial population is, the greater spatial momentum. 
(ii) $\mathrm{m}_{21}$, the rate of rural to urban migration. The higher the rate of migration to the urban region (which in this case is the same as the net rate of migration), the greater spatial momentum.

(iii) $\left(\rho_{2}-\rho_{1}\right)$, the extent to which the rural rate of natural increase exceeds the urban. The smaller $\left(\rho_{2}-\rho_{1}\right)$, the greater spatial momentum.

Element (iii) may seem counterintuitive, but an initial large difference in regional rates of natural increase can be expected to persist in the ultimate zero growth population. In that context, a relatively small urban rate of natural increase implies a lower ultimate proportion urban, thus less urbanization and a smaller $\Omega_{\mathrm{RR}}$. In our examination of hypothetical populations, we emphasize the role of these three elements in influencing spatial momentum.

\section{Decomposing the momentum of a multi-regional population}

To find the total momentum of a population, recognizing both age and region, one needs to reduce the initial fertility rates to replacement level, project the initial population to stationarity, and take the ratio of the ultimate to initial populations. The general population projection matrix to be considered is the $2 n$ by $2 n$ block Leslie matrix, $\mathbf{B}$, which applies to a population with two regions and $\mathrm{n}$ age groups and can be written as

$$
\mathbf{B}=\left[\begin{array}{ccccccc}
\mathrm{a}_{11} & \mathrm{a}_{12} & \mathrm{a}_{21} & \mathrm{a}_{22} & \ldots & \mathrm{a}_{\mathrm{n} 1} & \mathrm{a}_{\mathrm{n} 2} \\
\mathrm{~b}_{11} & \mathrm{~b}_{12} & \mathrm{~b}_{21} & \mathrm{~b}_{22} & \ldots & \mathrm{b}_{\mathrm{n} 1} & \mathrm{~b}_{\mathrm{n} 2} \\
\mathrm{~s}_{111} & \mathrm{~s}_{121} & 0 & 0 & \ldots & 0 & 0 \\
\mathrm{~s}_{112} & \mathrm{~s}_{122} & 0 & 0 & \ldots & 0 & 0 \\
0 & 0 & \mathrm{~s}_{211} & \mathrm{~s}_{221} & \ldots & 0 & 0 \\
0 & 0 & \mathrm{~s}_{212} & \mathrm{~s}_{222} & \ldots & 0 & 0 \\
\cdot & \cdot & 0 & 0 & \ldots & \cdot & \cdot \\
\cdot & \cdot & 0 & 0 & \ldots & . & \cdot \\
\cdot & . & \cdot & . & \ldots & . & \cdot
\end{array}\right]
$$

where $a_{i j}$ represents the rate for births in state 1 to persons age $i$ in state $j, b_{i j}$ represents the rate for births in state 2 to persons age $\mathrm{i}$ in state $\mathrm{j}$, and $\mathrm{s}_{\mathrm{kij}}$ represents the proportion 
of those age $\mathrm{k}$ in state $\mathrm{i}$ who survive to be in state $\mathrm{j}$ at age k+1 (cf. Keyfitz 1977: Chapter 2; Feeney 1970). When B represents a population's demographic behavior between times $t$ and $t+1$, we have the projection relationship

$$
\mathbf{p}_{\mathbf{t}+\mathbf{1}}=\mathbf{B} \mathbf{p}_{\mathbf{t}}
$$

where $\mathbf{p}_{\mathbf{t}}$ is the population vector at time $t$, whose odd elements, $p_{j 1}$, represent the number of persons age $\mathrm{j}$ in state 1 , and whose even elements, $\mathrm{p}_{\mathrm{j} 2}$, represent the number of persons age $\mathrm{j}$ in state 2 .

As is conventional, survivorship proportions $s$ reflect both mortality and migration, and after a move individuals immediately adopt the demographic behavior of their new state. That Markovian assumption is not likely to substantially affect the results. Empirically, adoption of the destination state's demographic patterns typically occurs over time. The Markovian assumption accelerates that process to greatly simplify the calculations.

There are many ways to reduce the elements of $\mathbf{B}$ to a stationary level, but what is sought is a way that is demographically plausible. In the single region model, a zero growth population projection matrix can be found by dividing every fertility rate by the population's Net Reproduction Rate (NRR). However, the NRR of a multiregional population is neither well defined nor easy to calculate. Simply dividing every element of $\mathbf{B}$ by $\lambda_{\mathrm{B}}$, the dominant eigenvalue of $\mathbf{B}$, is not appropriate because that would reduce survivorship and migration as well as fertility. A reasonable way to achieve stationarity is to follow Schoen and Kim (1996) and assume that fertility rates decline so that the new rates are given by

$$
\mathrm{c}_{\mathrm{ij}} *=\mathrm{c}_{\mathrm{ij}} / \lambda_{\mathrm{B}}^{\mathrm{i}}
$$

where the asterisk $\left(^{*}\right)$ indicates the stationary level rates and $\mathrm{c}$ refers to either the $\mathrm{a}$ or $\mathrm{b}$ elements of the first two rows of matrix $\mathbf{B}$. That transformation only affects fertility rates, preserves the original age-specific urban/rural fertility differentials, and is not difficult to implement. Most importantly, that transformation is consistent with the empirical pattern that fertility declines more at higher ages, produces reasonable agespecific patterns of fertility over a wide range of fertility levels, and depicts a pattern of change similar to that in the Coale-Trussell Model Fertility Schedules (Kim and Schoen 1996:22). Using transformed matrix $\mathbf{B}^{*}$, which is identical to $\mathbf{B}$ except that it has elements $\mathrm{c}^{*}$ in its first two rows, the initial population can be projected to stationarity and total momentum (denoted by $\Omega_{\mathrm{T}}$ ) determined. 
A natural way to find the age and region components of momentum is to collapse matrix $\mathbf{B}$ across age (or region) to produce the regional (or age) matrix that would be found if the other dimension had not been observed. The observed (or initial) population is used to weight the matrix elements in that process. Accordingly, let $\mathrm{n}$ by $\mathrm{n}$ Leslie matrix $\mathbf{A}$ be the age-specific population projection matrix obtained by collapsing $\mathbf{B}$ over region. With $\lambda_{\mathrm{A}}$ the dominant eigenvalue of $\mathbf{A}$, stationary matrix $\mathbf{A}^{*}$ can be obtained by dividing the ith first row element of $\mathbf{A}$ by $\lambda_{\mathrm{A}}{ }^{\mathrm{i}}$, a procedure that parallels the use of equation (8) in obtaining stationary matrix $\mathbf{B}^{*}$. Age momentum, denoted by $\Omega_{\mathrm{A}}$, can then readily be obtained by projecting the initial age-specific population to stationarity, and comparing its ultimate stationary size to the size of the initial population.

Now let 2 by 2 matrix $\mathbf{R}$, defined in equation (1), be the region-specific population projection matrix obtained by collapsing $\mathbf{B}$ over age. Again, the process weights the matrix elements by the observed population. Because age is not recognized in matrix $\mathbf{R}$, a stationary matrix cannot be found using the approach in equation (8). To find an appropriate procedure, consider the transfer rate matrix, $\boldsymbol{\mu}_{\mathbf{R}}$, shown in equation (2), that underlies $\mathbf{R}$. A desirable way to achieve stationarity is to reduce the regionspecific rates of natural increase while preserving the interregional transfer rates. As was implicitly done previously, that can be accomplished by subtracting the dominant eigenvalue of $\boldsymbol{\mu}_{\mathrm{R}}$, denoted by $r_{\mu}$, from both diagonal elements, yielding stationary rate matrix $\boldsymbol{\mu}_{\mathbf{R}}{ }^{*}=\boldsymbol{\mu}_{\mathbf{R}}-r_{\mu} \mathbf{I}$ (with $\mathbf{I}$ the 2 by 2 identity matrix). At the level of population projection matrix $\mathbf{R}=\exp \left[\boldsymbol{\mu}_{\mathbf{R}}\right]$, we can find stationary matrix $\mathbf{R}^{*}$ from the relationship

$$
\mathbf{R} * \mathbf{R} / \lambda_{\mathrm{R}}
$$

where $\lambda_{R}=\exp \left[r_{\mu}\right]$ represents the dominant eigenvalue of $\mathbf{R}$. Spatial momentum $\left(\Omega_{R}\right)$ can now be obtained by projecting the initial regional population to stationarity using $\mathbf{R}^{*}$, and finding $\Omega_{\mathrm{R}}$ by taking the ratio of the ultimate projected population to the initial population.

To complete the decomposition, an age-region interaction term is needed. Because momentum involves growth, an essentially multiplicative process, let us define the interaction momentum, $\Omega_{\mathrm{I}}$, by

$$
\Omega_{\mathrm{I}}=\Omega_{\mathrm{T}} /\left(\Omega_{\mathrm{A}} \Omega_{\mathrm{R}}\right)
$$


which yields the multiplicative relationship

$$
\Omega_{\mathrm{T}}=\Omega_{\mathrm{A}} \Omega_{\mathrm{R}} \Omega_{\mathrm{I}}
$$

Given equations (8) through (10), it is possible to take any age-regional population projection matrix B, find its total (age-region) momentum, and decompose that total momentum into components that reflect age momentum, spatial momentum, and the interaction between them.

\section{Numerical results from hypothetical populations}

Data are not readily available to calculate spatial momentum based on actual behavior in developing countries, where the proportions urban are relatively low. We therefore begin by examining the spatial momentum values that arise from assuming a range of plausible demographic inputs.

To simplify the calculations and make them more transparent, assumptions were made with regard to mortality, fertility, migration, and initial population composition. Fifteen year age groups are used throughout. Since mortality is peripheral to present concerns and world-wide mortality differentials have narrowed markedly, a single set of survivorship proportions are used. Given the low levels of mortality already observed in some developing countries [2], and anticipating future gains elsewhere, we assume a mortality schedule that roughly follows the Coale-Demeny West pattern and yields a life expectancy of about 78 years (see Appendix).

Fertility assumptions were made with regard to the level, age pattern, and size of the urban/rural differential. With regard to level, a "base" fertility pattern was established that put both urban and rural populations slightly above replacement level. Those fertility values (shown in the Appendix) are projection matrix elements that reflect the number of births who survive to the end of the projection interval in the state indicated for each person in the beginning population of the designated age and state. Initial fertility was characterized as either High or Medium. The Medium fertility scenario used rates 1.25 time the base values, and with a large urban/rural differential produced a stable growth rate of .0185 . The High fertility scenario used rates 1.8 times the base values, and produced a stable growth rate of .0340. Each fertility level was associated with a High and a Low urban/rural fertility differential. Under the High differential, rural rates were $40 \%$ higher than urban; under the Low differential, rural rates were 20\% higher (cf. Shapiro and Tambashe 1999).

Migration assumptions were based on patterns observed in more developed countries, in particular, those analyzed by Rogers and Castro (1986). As shown in the 
Appendix, a standard age pattern of migration was used that emphasized ages under 30, and the level of rural to urban migration was taken as twice the level of urban to rural migration. That ratio is consistent with rates reported for a number of developed countries during the 1970's (Rogers and Castro 1986:183-85, 189). That may be low for the contemporary developing world, but there are really no data on which to make a more accurate determination. Under the High migration scenario, $r_{21} / \lambda_{R}$ was .2 at ages 0-14 and .4 at ages 15-29; under the Medium migration scenario, $\mathrm{r}_{21} / \lambda_{\mathrm{R}}$ was .1 at ages 0-14 and .2 at ages 15-29. Some supplementary calculations, with lower relative urban to rural migration, were made with the under age 30 values of $r_{12} / \lambda_{R}$ set at a quarter rather than a half of $r_{21} / \lambda_{R}$.

The initial age composition of the population was chosen to approximate the age composition of the stable population implied by matrix $\mathbf{B}$. As shown in the Appendix, a different initial composition was used for each level of fertility and each initial percent urban. The initial percents urban used were chosen to approximate the urban proportion in the less developed areas of the world during 1970 and 1998 (UN Population Division 1999:26). The Low urban scenario used 25\% initially urban and the Medium scenario used $37.5 \%$. (Using the stable population percent urban is not appropriate because it is much higher than the proportion urban that is typically observed; moreover, it would yield a spatial momentum of zero.)

The above assumptions generated 2 fertility levels, 2 urban/rural fertility differentials, 2 levels of interregional migration, and 2 initial percents urban, for a total of 16 different models. Two additional scenarios were examined that had lower relative urban to rural migration, which was observed in some countries by Rogers and Castro (1986). The results are shown in Table 1, which presents the ultimate proportion urban and the four measures of momentum for each model.

Total momentum varied from 1.376 to 1.774 under Medium fertility and from 2.007 to 2.239 under High fertility. The value of $\Omega_{\mathrm{T}}$ declined when the initial percent urban increased, and when there was less interregional migration. When the urban/rural fertility differential decreased, $\Omega_{\mathrm{T}}$ generally (though not always) increased.

Age momentum was always the dominant component of $\Omega_{\mathrm{T}}$, and responded strongly to higher levels of fertility. Other effects were small, though a higher initial percent urban slightly lowered $\Omega_{\mathrm{A}}$ under Medium fertility while raising it somewhat more under High fertility.

Spatial momentum varied from a low of .95 to a high of 1.364 . It was quite sensitive to the difference between the initial and ultimate proportions urban, and consistently declined when the initial percent urban rose. Indeed, in most models, $\Omega_{R}$ was close to 1 plus the increase in proportion urban. Since the maximum ultimate 
Table 1: $\quad$ Momentum and Ultimate Proportion Urban Values for Hypothetical Populations by Fertility Level, Urban/Rural Fertility Differential, Initial Percent Urban, and Level of Urban/Rural Migration.

\begin{tabular}{|c|c|c|c|c|c|}
\hline \multirow[b]{2}{*}{$\begin{array}{l}\text { I. MEDIUM FERTILITY } \\
\text { A. High urban/rural fertility differential }\end{array}$} & \multicolumn{4}{|c|}{ Momentum } & \multirow{2}{*}{$\begin{array}{r}\text { Ultimate } \\
\text { Proportion } \\
\text { Urban }\end{array}$} \\
\hline & $\underline{\text { Total }}$ & Age & Spatial & Interaction & \\
\hline 1. $25 \%$ initially urban, high $u / r$ migration & 1.656 & 1.393 & 1.207 & .985 & .468 \\
\hline 2. $25 \%$ initially urban, medium $\mathrm{u} / \mathrm{r}$ migration & 1.572 & 1.393 & 1.094 & 1.032 & .337 \\
\hline 3. $37.5 \%$ initially urban, high $u / r$ migration & 1.478 & 1.345 & 1.084 & 1.013 & .462 \\
\hline 4. $37.5 \%$ initially urban, medium u/r migration & 1.376 & 1.345 & .950 & 1.076 & .329 \\
\hline \multicolumn{6}{|l|}{ B. Low urban/rural fertility differential } \\
\hline 1. $25 \%$ initially urban, high $\mathrm{u} / \mathrm{r}$ migration & 1.639 & 1.383 & 1.226 & .967 & .519 \\
\hline 2. $25 \%$ initially urban, medium u/r migration & 1.650 & 1.383 & 1.149 & 1.038 & .392 \\
\hline 3. $37.5 \%$ initially urban, high $u / r$ migration & 1.505 & 1.341 & 1.121 & 1.001 & .520 \\
\hline 4. $37.5 \%$ initially urban, medium $\mathrm{u} / \mathrm{r}$ migration & 1.493 & 1.341 & 1.019 & 1.092 & .394 \\
\hline \multicolumn{6}{|l|}{$\begin{array}{l}\text { C. Low urban/rural fertility differential, lower relative } \\
\text { urban to rural migration }\end{array}$} \\
\hline $\begin{array}{l}\text { 1. } 25 \% \text { initially urban, high migration to urban } \\
\text { region }\end{array}$ & 1.774 & 1.388 & 1.364 & .937 & .599 \\
\hline $\begin{array}{l}\text { 2. } 37.5 \% \text { initially urban, high migration to urban } \\
\text { region }\end{array}$ & 1.612 & 1.341 & 1.231 & .976 & .590 \\
\hline \multicolumn{6}{|l|}{ II. HIGH FERTILITY } \\
\hline \multicolumn{6}{|l|}{ A. High urban/rural fertility differential } \\
\hline 1. $25 \%$ initially urban, high $u / r$ migration & 2.235 & 1.901 & 1.190 & .988 & .451 \\
\hline 2. $25 \%$ initially urban, medium $\mathrm{u} / \mathrm{r}$ migration & 2.115 & 1.901 & 1.089 & 1.021 & .333 \\
\hline 3. $37.5 \%$ initially urban, high $u / r$ migration & 2.159 & 2.008 & 1.059 & 1.015 & .437 \\
\hline 4. $37.5 \%$ initially urban, medium $u / r$ migration & 2.007 & 2.008 & .941 & 1.062 & .320 \\
\hline \multicolumn{6}{|l|}{ B. Low urban/rural fertility differential } \\
\hline 1. $25 \%$ initially urban, high $u / r$ migration & 2.239 & 1.883 & 1.210 & .983 & .530 \\
\hline 2. $25 \%$ initially urban, medium $\mathrm{u} / \mathrm{r}$ migration & 2.247 & 1.883 & 1.170 & 1.020 & .423 \\
\hline 3. $37.5 \%$ initially urban, high $\mathrm{u} / \mathrm{r}$ migration & 2.223 & 1.988 & 1.108 & 1.009 & .514 \\
\hline 4. $37.5 \%$ initially urban, medium $u / r$ migration & 2.188 & 1.988 & 1.030 & 1.068 & .405 \\
\hline
\end{tabular}


percent urban in all of the models in Table 1 was only $60 \%$, the scenarios considered are quite conservative with regard to the long term proportion urban. Spatial momentum declined markedly as the level of interregional migration fell. The value of $\Omega_{R}$ also responded to changes in the relative size of the migration rates; when the urban to rural rate went from $1 / 2$ to $1 / 4$ of the rural to urban rate, spatial momentum increased by more than .1. As expected from equation (5), $\Omega_{R}$ increased modestly when the urban/rural fertility differential declined. In most cases, there was little age-region interaction, as $\Omega_{\mathrm{R}}$ was quite insensitive to the level of fertility.

\section{Spatial momentum in Mexico, 1970}

Mexico is a country where the demographic transition began around 1970, and which has experienced substantial internal and international migration. Using data from the U.S. Census Bureau International Data Base (www.census.gov/ipc/www/idbprint.html), it is possible to estimate the urban/rural population projection matrix that characterized the female population of Mexico in 1970. The Census Bureau data provide 1970 female population data by age and urban/rural residence (Table 004), female deaths by age and urban/rural residence (Table 011), birth rates by age of mother (Table 028), and female net migrants by age and urban/rural residence (Table 036). Births in 1970 were allocated to urban and rural areas based on the urban/rural proportions of 1969 births in the Demographic Yearbook 1973 (United Nations 1974:227). Since the data only provide numbers of net migrants, the rural to urban and urban to rural levels were estimated based on flows to and from capital cities shown in Rogers and Castro (1986:184), with the assumed pattern emphasizing flows from rural to urban.

Table 2 shows the estimated urban/rural population projection (B) matrix for Mexican females in 1970, the initial and ultimate proportions urban, and the calculated momentum values. Total momentum was quite large (2.031), with age momentum again the dominant component (1.721). Although the percent urban only increased from $60 \%$ to $82 \%$, spatial momentum was substantial (1.147). That figure is well within the range shown in Table 1 , and indicates that in the context of an actual developing country, rural to urban migration and the urban/rural fertility differential can combine to significantly increase population growth during a transition to stationarity. 
Table 2: $\quad$ Estimated Urban/Rural Population Projection (B) Matrix, Initial and Ultimate Proportions Urban, and Momentum Values for Mexico, 1970 (Females)

\begin{tabular}{|c|c|c|c|c|c|c|c|c|c|c|c|}
\hline & $\begin{array}{r}\text { Region } \\
\text { Age }\end{array}$ & $\begin{array}{l}\text { Urban } \\
0-14\end{array}$ & $\begin{array}{l}\text { Rural } \\
0-14\end{array}$ & $\begin{array}{l}\text { Urban } \\
15-29\end{array}$ & $\begin{array}{l}\text { Rural } \\
15-29\end{array}$ & $\begin{array}{l}\text { Urban } \\
30-44\end{array}$ & $\begin{array}{l}\text { Rural } \\
30-44\end{array}$ & $\begin{array}{l}\text { Urban } \\
45-59\end{array}$ & $\begin{array}{l}\text { Rural } \\
45-59\end{array}$ & $\begin{array}{l}\text { Urban } \\
60-74\end{array}$ & $\begin{array}{l}\text { Rural } \\
60-74\end{array}$ \\
\hline \multicolumn{12}{|c|}{ Fertility: } \\
\hline & Urban & 0.6725 & 0.0780 & 1.0926 & 0.2947 & 0.6617 & 0.1123 & & & & \\
\hline & Rural & 0.0108 & 0.6966 & 0.0435 & 1.5967 & 0.0136 & 0.6529 & & & & \\
\hline \multicolumn{12}{|c|}{$\frac{\text { Survivorship }}{\text { to Ages: }}$} \\
\hline Urban & $15-29$ & 0.8618 & 0.2160 & & & & & & & & \\
\hline Rural & $15-29$ & 0.0255 & 0.6365 & & & & & & & & \\
\hline Urban & $30-44$ & & & 0.9211 & 0.4175 & & & & & & \\
\hline Rural & $30-44$ & & & 0.0523 & 0.5480 & & & & & & \\
\hline Urban & $45-59$ & & & & & 0.9106 & 0.3244 & & & & \\
\hline Rural & $45-59$ & & & & & 0.0334 & 0.6028 & & & & \\
\hline Urban & $60-74$ & & & & & & & 0.8652 & 0.2561 & & \\
\hline Rural & $60-74$ & & & & & & & 0.0160 & 0.5912 & & \\
\hline Urban & $75-89$ & & & & & & & & & 0.4579 & 0.1027 \\
\hline Rural & $75-89$ & & & & & & & & & 0.0066 & 0.3281 \\
\hline
\end{tabular}

Proportion Urban

Initial $\quad .596$

Ultimate $\quad .820$

Momentum

Total

2.031

Age

1.721

Spatial

1.148

Interaction

1.028 


\section{Summary and conclusions}

Although largely neglected, spatial momentum is an intrinsic part of population dynamics, and is a phenomenon inherent in the demographic patterns prevailing in the developing and urbanizing areas of the world. Spatial momentum is greater when the initial proportion urban is low, when there is substantial net rural to urban migration, and when the difference between the rural and urban rates of natural increase is modest. Calculations are presented for the population of Mexico, 1970 and for some 18 hypothetical populations that reflect a range of plausible assumptions regarding urban and rural growth and interregional migration. The results indicate that spatial momentum has an appreciable effect above and beyond age momentum. Third World proportions urban are still relatively low, net rural to urban migration continues to be a prominent feature of developing country demographic behavior, and urban/rural fertility differentials remain substantial. Spatial momentum is thus a second demographic phenomenon that is likely to significantly increase future world population growth.

\section{Acknowledgments}

Research assistance was provided by Stefan Jonsson and Nicola Standish, and the work benefited from comments by Young J. Kim, Michael Rendall, and Kenneth Wachter. Support under grant R01 HD28443 from the Center for Population Research (NICHD) is gratefully acknowledged. 


\section{Notes}

1. The pattern of higher rates of natural increase in rural areas continues to characterize developing countries. Data in the 1997 Demographic Yearbook (United Nations 1999: Tables 9 and 18) allow urban and rural rates of natural increase to be calculated for 12 countries: Mauritius 1996, Cuba 1995, Mexico 1995, Chile 1996, Peru 1993, Suriname 1996, Georgia 1995, Iran 1994, Kazakhstan 1996, Kyrgyzstan 1995, Tajikistan 1994, and Uzbekistan 1994. Rural rates of natural increase were higher in 10 of the 12 . The two exceptions, Chile 1996 and Georgia 1995, had relatively low fertility in both regions, with urban areas having the higher crude birth rates. Only 4 of the 12 (Chile 1996, Peru 1993, Georgia 1995, and Iran 1994) had higher rural than urban crude death rates.

2. According to figures in the 1997 Demographic Yearbook (United Nations 1999: Table 22), female life expectancy at birth was 77.6 years in Costa Rica 1990-95, 77.3 years in Cuba 1990-95, and 78.2 years in Iraq 1990. 


\section{References}

Bongaarts, J. and R.A. Bulatao. 1999. Completing the demographic transition. Population and Development Review 25:515-29.

Brockerhoff, M. 1999. Urban growth in developing countries: a review of projections and predictions. Population and Development Review 25:757-78.

Caswell, H. 1989. Matrix Population Models. Sunderland MA: Sinauer Associates.

Feeney, G.M. 1970. Stable age by region distributions. Demography 7:341-48.

Keyfitz, N. 1971. On the momentum of population growth. Demography 8:71-80.

Keyfitz, N. 1977. Introduction to the Mathematics of Population (2d Ed) Reading MA: Addison-Wesley.

Kim, Y.J. and R. Schoen. 1993. Crossovers that link populations with the same vital rates. Mathematical Population Studies 4:1-19.

Kim, Y.J. and R. Schoen. 1996. Populations with quadratic exponential growth. Mathematical Population Studies 6:19-33.

Kim, Y.J. and R. Schoen. 1997. Population momentum expresses population aging. Demography 34: 421-27.

Kim. Y.J., R. Schoen, and P.S. Sarma. 1991. Momentum and the growth-free segment of a population. Demography 28: 159-73.

Preston, S.H. 1986. The relation between actual and intrinsic growth rates. Population Studies 40:343-51.

Rogers, A. and L.J. Castro. 1986. Migration. Chapter 5 in A. Rogers and F.J. Willekens (Eds.) Migration and Settlement: A Multiregional Comparative Study. Dordrecht: Reidel.

Rogers, A. and F. Willekens. 1978. The spatial reproductive value and the spatial momentum of zero population growth. Environment and Planning A 10:503-18.

Schoen, R. 1988. Modeling Multigroup Populations. New York: Plenum.

Schoen, R. and Y.J. Kim. 1993. Two-state spatial dynamics in the absence of age. Theoretical Population Biology 44:67-79.

Schoen, R. and Y.J. Kim. 1996. Stabilization, birth waves, and the surge in the elderly. Mathematical Population Studies 6:35-53. 
Shapiro, D. and B.O. Tambashe. 1999. Fertility transition in urban and rural areas of sub-Saharan Africa. Paper presented at the 1999 Chaire Quetelet Symposium in Demography, Catholic University of Louvain, Belgium.

United Nations, Dept of Economic and Social Affairs. 1974. Demographic Yearbook 1973. New York: United Nations.

United Nations, Dept of Economic and Social Affairs. 1999. Demographic Yearbook 1997. New York: United Nations.

United Nations, Population Division. 1999. Population Growth, Structure and Distribution: The Concise Report. New York: United Nations.

U.S. Bureau of the Census, International Data Base. From website at www.census.gov/ipc/www/idbprint.html

Wachter, K.W. 1988. Age group growth rates and population momentum. Population Studies 42:487-94. 


\section{Appendix}

\begin{tabular}{|c|c|c|c|c|c|c|}
\hline \multirow[t]{3}{*}{ Age } & \multirow{3}{*}{$\begin{array}{c}15 \text { Year } \\
\text { Survivorship } \\
\text { Proportion }\end{array}$} & \multicolumn{5}{|c|}{ Base Fertility Schedule } \\
\hline & & $\begin{array}{l}\text { Urban to } \\
\text { Urban }\end{array}$ & $\begin{array}{l}\text { Rural to } \\
\text { Urban }\end{array}$ & $\begin{array}{l}\text { Urban to } \\
\text { Rural }\end{array}$ & & \\
\hline & & & & & $\begin{array}{c}\text { Low } \\
\text { Differential }\end{array}$ & $\begin{array}{l}\text { High } \\
\text { Differential }\end{array}$ \\
\hline $0-14$ & .99 & .3 & .04 & .020 & .40 & .5 \\
\hline $15-29$ & .99 & .6 & .05 & .025 & .65 & .7 \\
\hline $30-44$ & .96 & .1 & .01 & .005 & .15 & .2 \\
\hline $45-59$ & .91 & & & & & \\
\hline $60-74$ & .51 & & & & & \\
\hline $75-89$ & --- & & & & & \\
\hline
\end{tabular}

\begin{tabular}{llllll}
\hline \multirow{2}{*}{ Age } & \multicolumn{2}{l}{$\begin{array}{l}\text { Proportion experiencing } \\
\text { rural to urban migration }\end{array}$} & \multicolumn{2}{l}{ Proportion experiencing urban to rural migration } \\
& High & Medium & High (Standard) & High (lower relative) & Medium \\
\hline $0-14$ & .2 & .1 & .1 & .05 & .05 \\
$15-29$ & .4 & .2 & .2 & .1 & .1 \\
$30-44$ & .01 & .01 & .01 & .01 & .01 \\
$45-59$ & .01 & .01 & .01 & .01 & .01 \\
$60-74$ & .01 & .01 & .01 & .01 & .01 \\
$75-89$ & --- & --- & --- & -- & -- \\
\hline
\end{tabular}


Demographic Research - Volume 6, Article 3

\begin{tabular}{|c|c|c|c|c|c|c|c|c|}
\hline \multicolumn{9}{|c|}{ POPULATION DISTRIBUTION (Total population=17.6) } \\
\hline \multirow[t]{3}{*}{ Age } & \multicolumn{4}{|c|}{ Medium Fertility } & \multicolumn{4}{|c|}{ High Fertility } \\
\hline & \multicolumn{2}{|c|}{ Initially $25 \%$ Urban } & \multicolumn{2}{|c|}{$\begin{array}{l}\text { Initially } 37.5 \% \\
\text { Urban }\end{array}$} & \multicolumn{2}{|c|}{ Initially $25 \%$ Urban } & \multicolumn{2}{|c|}{$\begin{array}{l}\text { Initially } 37.5 \% \\
\text { Urban }\end{array}$} \\
\hline & Urban & Rural & Urban & Rural & Urban & Rural & Urban & Rural \\
\hline $0-14$ & 1.0 & 4.2 & 1.5 & 3.5 & 1.3 & 6.2 & 2.3 & 5.8 \\
\hline $15-29$ & .8 & 3.2 & 1.2 & 2.7 & 1.1 & 3.5 & 1.7 & 2.9 \\
\hline $30-44$ & .9 & 2.3 & 1.3 & 1.9 & 1.0 & 1.6 & 1.2 & 1.1 \\
\hline $45-59$ & .8 & 1.7 & 1.2 & 1.4 & .6 & 1.0 & .7 & .7 \\
\hline $60-74$ & .6 & 1.2 & .9 & 1.0 & .3 & .6 & .5 & .4 \\
\hline $75-89$ & .3 & .6 & .5 & .5 & .1 & .3 & .2 & .1 \\
\hline Total & 4.4 & 13.2 & 6.6 & 11.0 & 4.4 & 13.2 & 6.6 & 11.0 \\
\hline
\end{tabular}

